\title{
Identification and quantification of novel RNA isoforms in horn cancer of Bos indicus by comprehensive RNA-Seq
}

\author{
Subhash J. Jakhesara ${ }^{1} \cdot$ Prakash G. Koringa $^{1} \cdot$ Neelam M. Nathani $^{1} \cdot$ \\ Chaitanya G. Joshi ${ }^{1}$
}

Received: 17 October 2016/Accepted: 26 November 2016/Published online: 7 December 2016

(C) The Author(s) 2016. This article is published with open access at Springerlink.com

\begin{abstract}
Horn cancer (HC) is a squamous cell carcinoma of horn, commonly observed in Bos indicus of the Asian countries. To elucidate the complexity of alternative splicing present in the $\mathrm{HC}$, high-throughput sequencing and analysis of $\mathrm{HC}$ and matching horn normal $(\mathrm{HN})$ tissue were carried out. A total of 535,067 and 849,077 reads were analysed after stringent quality filtering for $\mathrm{HN}$ and $\mathrm{HC}$, respectively. Cufflinks pipeline for transcriptome analysis revealed 4786 novel splice isoforms comprising 2432 exclusively in HC, 2055 exclusively in $\mathrm{HN}$ and 298 in both the conditions. Based on pathway clustering and in silico verification, 102 novel splice isoforms were selected and further analysed with respect to change in protein sequence using Blastp. Finally, fourteen novel splicing events supported both by Cufflinks and UCSC genome browser were selected and confirmed expression by RT-qPCR. Future studies targeted at in-depth characterization of these potential candidate splice isoforms might be helpful in the development of relevant biomarkers for early diagnosis of HC. The results reported in this study refine the available information on transcriptome repertoire of bovine species and boost the research in the line of development of relevant biomarkers for early diagnosis of $\mathrm{HC}$.
\end{abstract}

Electronic supplementary material The online version of this article (doi:10.1007/s13205-016-0577-5) contains supplementary material, which is available to authorized users.

Subhash J. Jakhesara

drsubhash81@gmail.com

1 Department of Animal Biotechnology, College of Veterinary Science and Animal Husbandry, Anand Agricultural University, Anand, Gujarat 388 001, India
Keywords Horn cancer - Alternative splicing · GS-FLX Titanium $\cdot$ Transcriptome profiling $\cdot$ Splice isoform

$\begin{array}{ll}\text { Abbreviations } \\ \text { HC } & \text { Horn cancer } \\ \text { HN } & \text { Horn normal } \\ \text { AS } & \text { Alternative splicing } \\ \text { EST } & \text { Expressed sequence tag } \\ \text { KEGG } & \text { Kyoto encyclopedia of genes and genomes } \\ \text { DAVID } & \begin{array}{l}\text { Database for annotation, visualization and } \\ \text { integrated discovery }\end{array} \\ \text { BLAT } & \text { Blast-like alignment tool } \\ \text { IGV } & \text { Integrated genome viewer } \\ \text { GO } & \text { Gene ontology } \\ \text { NMD } & \text { Nonsense-mediated decay }\end{array}$

\section{Introduction}

Cancer is a multifactorial disease affecting almost every species of animals and human. Various types of cancers are prevalent in farm animals causing moderate to severe economic losses. Horn cancer (HC, cancerous tissue from affected horn) is amongst widely prevalent cancer reported in Indian zebu cattle (Bos indicus), drawing recent attention towards the necessity of in-depth studies for better understanding. $\mathrm{HC}$ is a progressive cancer which is classified into three different stages based on the growth of tumour tissue in the affected horn. The incidence of HC in India is more commonly seen in Kankrej breed than other zebu and crossbred cattle (Joshi et al. 2009; Udharwar et al. 2008). Exceptional few cases of $\mathrm{HC}$ have been reported from other countries like Sumatra (Burggraaf 1935), Brazil

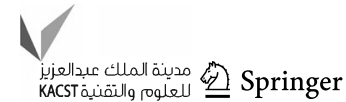


(Rezende and Naves 1975) and Iraq (Zubaidy 1976). The mainly affected animals are Bullocks $(95 \%)$ in comparison to cows $(5 \%)$, whereas it is rarely reported in bulls, buffaloes, sheep and goats (Chattopadhyay et al. 1982; Damodaran et al. 1979; Gupta et al. 1980; Kulkarni 1953). $\mathrm{HC}$ is a squamous cell carcinoma of horn arising from pseudo-stratified columnar epithelium of the horn core mucosa. The main difficulties in early diagnosis include the absence of specific markers and the lack of a complete understanding of the cellular hierarchy of morphogenesis of the horn core epithelium. At molecular level, HC has been studied previously for novel gene content (Jakhesara et al. 2013b) and global gene expression (Tripathi et al. 2012).

Horn cancer involves complex interplay of several genes which are up-regulated or down-regulated and results in the transformation of normal horn tissue to malignant one. In horn cancer, the interplay of three genes KRT6A, KRT6B, and $K R T 6 C$ has been observed in cancer. Over and above these keratin protein family members, there is also upregulation of a novel candidate KRT84. Other genes of keratin family, KRT14 and KRT5, are specifically expressed in the basal layer of the epidermis and found to be upregulated in HC. Gene KRT14 expression can be considered as a candidate of proliferative activity and metastatic potential of SCC in horn. Besides keratin family genes, Stratifin (SFN), Anilin (ANLN), NR4Al and SCGBlAl (Uteroglobin) were also significantly up-regulated in $\mathrm{HC}$ tissue. While, a number of genes were observed to be down-regulated in HC including CXCL17, KRT19 and $B P I F B 1$ which are known to be involved in anti-tumour immune reactions. $C X C L 17$ has also been reported to have potential tumour suppressor activity (Hiraoka et al. 2011). $B P I F B 1$ is commonly known as long palate, lung and nasal epithelium carcinoma-associated protein 1 (LPLUNC1) expressed in the epithelium of upper respiratory tract (Bingle et al. 2010). Additional details about commonly expressed genes in $\mathrm{HC}$ are described elsewhere (Koringa et al. 2013).

Amongst diverse type of changes present in the cancer, overrepresentation of aberrant mRNA transcripts is the most common and widely reported observation (Jakhesara et al. 2013a; Li et al. 2013; Erho et al. 2012). Alternative splicing (AS) is the phenomenon by which the exons of primary transcripts are used alternatively to produce structurally and functionally distinct mRNA (splice isoforms) and protein variants, which might be one of the most extensively used mechanisms that accounts for the greater cellular complexity of higher eukaryotic organisms (Irimia and Blencowe 2012). Cancer cells may exhibit defective regulation of this alternative exon usage that can result in changes with potential transcriptomewide consequences to gene function. It is imperative that
AS can result in increased protein repertoire of the cell encoding alternative protein forms with biological functions that differ from the canonical product of the locus. AS transcripts exhibit changes to the primary transcript and might result in transcripts and/or protein isoforms with loss or disruption of domains responsible for original function.

Detailed investigation and understanding of complexity of AS events exhibited by cancer cells are very important as it provides important clues about structural and functional consequences of deregulated genes. Until recently, profound cost, limited coverage and sensitivity of the sequencing and microarray studies were hindering the global study of AS events in the cell; however, with advancement in the next-generation sequencing technologies now it is possible to do the same. Although, $\mathrm{HC}$ has been studied recently for detection of AS events, the information generated was on limited scale, with small size of dataset and analysis lacking RNA-Seq-specific pipeline (Patel et al. 2013). In the present study, we employed highthroughput pyro sequencing combined with Cufflinks workflow optimized for RNA-Seq to elucidate complexity of the AS in HC transcriptome. Here, we report AS events exhibited in $\mathrm{HC}$ and $\mathrm{HN}$ tissue and confirmed their significant association with HC by RT-qPCR.

\section{Materials and methods}

\section{Tissue collection and RNA sequencing}

All experimental protocols were performed as per guidelines and approval of Institutional Animal Ethics Committee of the Anand Agricultural University (Permit Number 486). Detailed summary of methods employed for tissue collection and sequencing is described elsewhere (Jakhesara et al. 2013b). Briefly, after mRNA isolation from collected tissues of $\mathrm{HC}$ and $\mathrm{HN}$, cDNA libraries were prepared and sequenced on GS FLX Titanium following manufacturer protocol (Roche Diagnostics, Switzerland).

\section{Mapping of RNA-Seq reads using GMAP}

After signal processing and trimming, available reads were aligned to the UCSC Bos taurus reference genome (build bosTau7) using GMAP v2012-06-06 (Wu and Watanabe 2005). Whenever required, we used SAM Tools v0.1.18 ( $\mathrm{Li}$ et al. 2009) to convert SAM files to BAM file and vice versa. Mapping results were visualized using a local copy of the Integrative Genomics Viewer v 2.1.17 (Thorvaldsdottir et al. 2012) software available at http://www. broadinstitute.org/igv/. 


\section{Transcript assembly using Cufflinks}

The read alignments from GMAP were processed by Cufflinks v2.0.0 (Trapnell et al. 2010) for assembly of the reads into transcripts; their abundance estimation and tests for differential expression between the cancer and normal tissue samples were performed using Cufflinks. Cufflinks also uses reference annotation-based transcript (RABT) assembly method (Roberts et al. 2011), to assemble against a known reference annotation to better identify novel transcripts. Cufflinks measures transcript abundances in Fragments Per Kilobase of exon per Million fragments mapped (FPKM), which is analogous to single-read "RPKM". Cufflinks was run with default parameters for RABT assembly method.

\section{Comparison to reference annotation and differential analysis}

Once assembly of alignments of reads to transcripts is completed; the transcripts.gtf files from Cufflinks were used as input to Cuffcompare along with reference annotation file refFlat.gtf file downloaded from UCSC Genome Browser Database (build Btau7) (Dreszer et al. 2012). Combined.gtf file produced by Cuffcompare was provided as input to Cuffdiff along with alignment files produced by GMAP for differential analysis between two samples. Cuffcompare and Cuffdiff were run employing default parameters with refFlat.gtf file of Bos taurus genome Btau7 build for reference annotation.

\section{Functional analysis of gene isoforms}

The Database for Annotation, Visualization and Integrated Discovery (DAVID) v6.7 is a set of web-based functional annotation tools for functional clustering of genes (Huang et al. 2009). The functional clustering tool was used for functional enrichment of isoforms with significant differential expression. Gene ontology was selected for functional annotation with Fishers exact test at threshold value of 0.001. Similarly, pathway analysis is also performed with DAVID employing Fishers exact test at a threshold value of 0.01 .

\section{Validation of potential novel splices isoforms}

The tracking file provided by Cuffcompare was used to infer splice isoforms unique to cancer by examining manually. The transcripts with class code $j$ were selected for further evaluation. All novel isoforms detected by Cuffcompare were classified based on their uniqueness in case of $\mathrm{HC}, \mathrm{HN}$ and present in both the cases. Further, these novel isoforms were subjected to pathway-based clustering using the highest classification stringency parameter in DAVID v6.7. From these, novel splice isoforms clustered under pathways associated with cancer were selected for further in silico verification with IGV for actual read support and BLAT program of UCSC genome browser for EST support. Transcripts assembled from the reads supporting these splice isoforms were translated into protein sequence for evaluation of protein change by comparison with reference protein sequence and loss of functional domain by blastp (Altschul et al. 1990). At last, fourteen transcripts showed novel splicing both by Cufflinks and UCSC genome browser and a significant change in protein sequence with loss of functional motif was confirmed by RT-PCR. The primers for RT-PCR were designed from exon-exon junction in such a way that it should flank the location of splice site change.

\section{Quantitative real-time PCR (qPCR)}

Four samples each of $\mathrm{HC}$ and $\mathrm{HN}$ were used to isolate total RNA and differential expression of fourteen novel splice isoforms was confirmed through RT-qPCR. The FASTA sequences of reads spanning each selected transcript were obtained from IGV and queried using web based tool Primer-BLAST (Ye et al. 2012) against Bos taurus reference transcriptome to check the specificity of primers to amplify only isoform transcripts. The expression level of novel splice isoforms was quantified by RT-qPCR analysis on ABI PRISM 7500 fast real-time PCR system (Applied Biosystems) using Quantifast SYBR Green PCR master mix (Qiagen). For PCR, all reactions were run in triplicate and cycle threshold $\left(C_{\mathrm{T}}\right)$ values for target novel transcripts were normalized with endogenous reference gene RPLP0. The fold change between two conditions was calculated using the formula as described here:

Fold expression $=2^{-\Delta \Delta C_{\mathrm{T}}}$

where $\Delta C_{\mathrm{T}}$ is the average $C_{\mathrm{T}}$ of target isoform - average $C_{\mathrm{T}}$ of endogenous control (RPLP0), and $\Delta \Delta C_{\mathrm{T}}$ is the $\Delta C_{\mathrm{T}}$ of target sample (HC) $-\Delta C_{\mathrm{T}}$ of calibrator sample $(\mathrm{HN})$.

\section{Results}

\section{Deep sequencing of Bos indicus horn transcriptome}

To fully characterize and identify $\mathrm{HC}$ transcriptome, three technical replicates of $\mathrm{HC}$ and $\mathrm{HN}$ sample were processed for sequencing in three different runs. Thereafter, we pooled sequencing data from all three technical replicates of each sample for further analysis. As a result of sequencing, we obtained a total of 0.59 million and 0.92 million high-quality reads comprising 239 million and 372 
million nucleotide bases for $\mathrm{HN}$ and $\mathrm{HC}$ tissue, respectively. The reads used for analysis are submitted to the bioProject NCBI and publically available with Accession number PRJNA353983. Raw sequenced reads were processed for quality filtering on the basis of quality score and homopolymer runs. After quality filtering, we retained 535,067 and 849,077 reads, respectively, for $\mathrm{HN}$ and $\mathrm{HC}$, for further mapping and analysis of digital gene expression profiling. We used GMAP software package for alignment of sequencing sequenced reads against Bos taurus reference genome (bTau7). Approximately, 94.8 and $94.3 \%$ of reads were successfully mapped to reference consisting of 80.3 and $79.7 \%$ of uniquely mapped reads, respectively, for $\mathrm{HC}$ and HN. Detailed summary of mapping is described elsewhere (Jakhesara et al. 2013b).

\section{Differentially expressed gene isoforms}

In total, 13,020 known isoforms were detected in $\mathrm{HC}$ while 12,728 known isoforms were detected in HN. Amongst these, 2739 isoforms were detected only in $\mathrm{HC}$, while 2447 isoforms were detected only in HN. In total, 1252 known isoforms were up-regulated, while 1543 down-regulated isoforms were observed with at least twofold difference in HC tissue in comparison to HN. Top ten up- and downregulated isoforms with significant differential expression are presented in Fig. 1a. More extensive lists of all 130 gene isoforms with significant differential expression in HC are given in Table ST1. The fold change amongst top 50 up-regulated isoforms was from 11 to 2.7 ; while in case of down-regulated isoforms, it was -8 to -2.5 -fold.

\section{Functional annotation of isoforms with significant differential expression}

All isoforms with differential expression were subjected to functional analyses for gene ontology and pathway analysis. The results revealed significant enrichment of cancer (9.63E-04) and colorectal cancer $(5.80 \mathrm{E}-03)$ pathways. Gene ontology analyses also revealed enrichment of several biological processes at threshold of 0.001. Mainly, biological processes associated with angiogenesis, epithelial cell development and differentiation, cell proliferation and cell death were found to be enriched in differentially expressed genes. Table 1 shows list of biological processes and pathways enriched in differentially expressed isoforms.

\section{Novel splice isoforms and their validation}

Cuffcompare detected several transcripts with novel splicing and all detected transcripts were classified as intergenic, complete match, novel or unknown. A total of 4785 novel splice isoforms were detected by Cuffcompare, which included 2432 isoforms unique for HC, 2055 isoforms unique for $\mathrm{HN}$ and 298 splice isoforms were observed in both the conditions. Figure $1 \mathrm{~b}$ shows top 10 novel up- and down-regulated splice isoforms in $\mathrm{HC}$ and HN sample.

The large number of novel splice isoforms was found in the present study; hence, to select the events relevant to the cancer, KEGG pathway analyses and gene ontology analyses were performed. Splice isoforms uniquely present in $\mathrm{HC}$ and $\mathrm{HN}$ as well as common isoforms in both conditions were subjected to KEGG pathway analyses using DAVID tool employing highest sensitivity. As a result of this analysis, three clusters (cancer-related pathways, cardiomyopathy and amino acid metabolism) were found to be enriched in $\mathrm{HC}$ and $\mathrm{HN}$. Based on this analysis and in silico verification with IGV, respectively, 42, 43 and 17 splice isoforms were selected for $\mathrm{HC}, \mathrm{HN}$ and common to both conditions (Table ST2). Figure 2 shows representative image of one such novel splice isoform detected in FOS gene.

All splice isoforms were evaluated with blastp for protein change and details are available as Table ST2. Finally, 14 transcripts (7 isoforms uniquely expressed in $\mathrm{HC}, 5$ transcripts uniquely expressed in $\mathrm{HN}$ and 2 isoforms present in both) were selected for RT-qPCR verification based on isoforms showing frame shift, loss of active domain and association with cancer. Table 2 shows list of splice isoforms with type of variation selected for RT-qPCR. Primers designed from exon-exon junction for amplification of these reference and variant transcript are given in Table ST3.

The expression profile of 14 novel splice events and the corresponding reference transcripts obtained with RTqPCR is presented in Fig. 3. The RT-qPCR analysis of HC and $\mathrm{HN}$ tissue confirmed the expression of all reference as well as novel splice isoforms except FGFR2 isoform, which could not be amplified after repeated attempts on different samples. However, we found some discrepancies between RT-qPCR and RNA-Seq expression results. RTqPCR revealed down-regulation of all the splice isoforms which were found to be up-regulated by RNA-Seq except MAPK9, while in case of down-regulated isoforms complete correlation was observed between RT-qPCR and RNA-Seq.

\section{Discussion}

Cancer results from culmination of variety of genetic and epigenetic changes occurring over a period of time. In such a disease, it would be very much helpful to examine molecular signatures that differentiate normal cell from cancer one, as it will unravel nature and pathologic behaviour of a cancer cell. Here, we have made an attempt to 
Fig. 1 Top 10 up-/downregulated isoforms in horn cancer. a Top 10 up-/downregulated isoforms with significant differential expression in HC. The differentially expressed isoforms in $\mathrm{HC}$ vs. those in normal tissue were determined by Cuffdiff. The fold change is the ratio of FPKM of these isoforms in $\mathrm{HC}$ to FPKM in normal tissue. The differentially expressed isoforms were ranked on their fold change and 10 isoforms with the highest or lowest fold changes are shown in the figure. b Top 10 up-/down-regulated novel splice variants in HC. The fold change is the ratio of FPKM of these isoforms in $\mathrm{HC}$ to FPKM in normal tissue. The novel splice variants were ranked on their fold change and 10 splice variants with the highest or lowest fold changes are shown here (a) 15 -
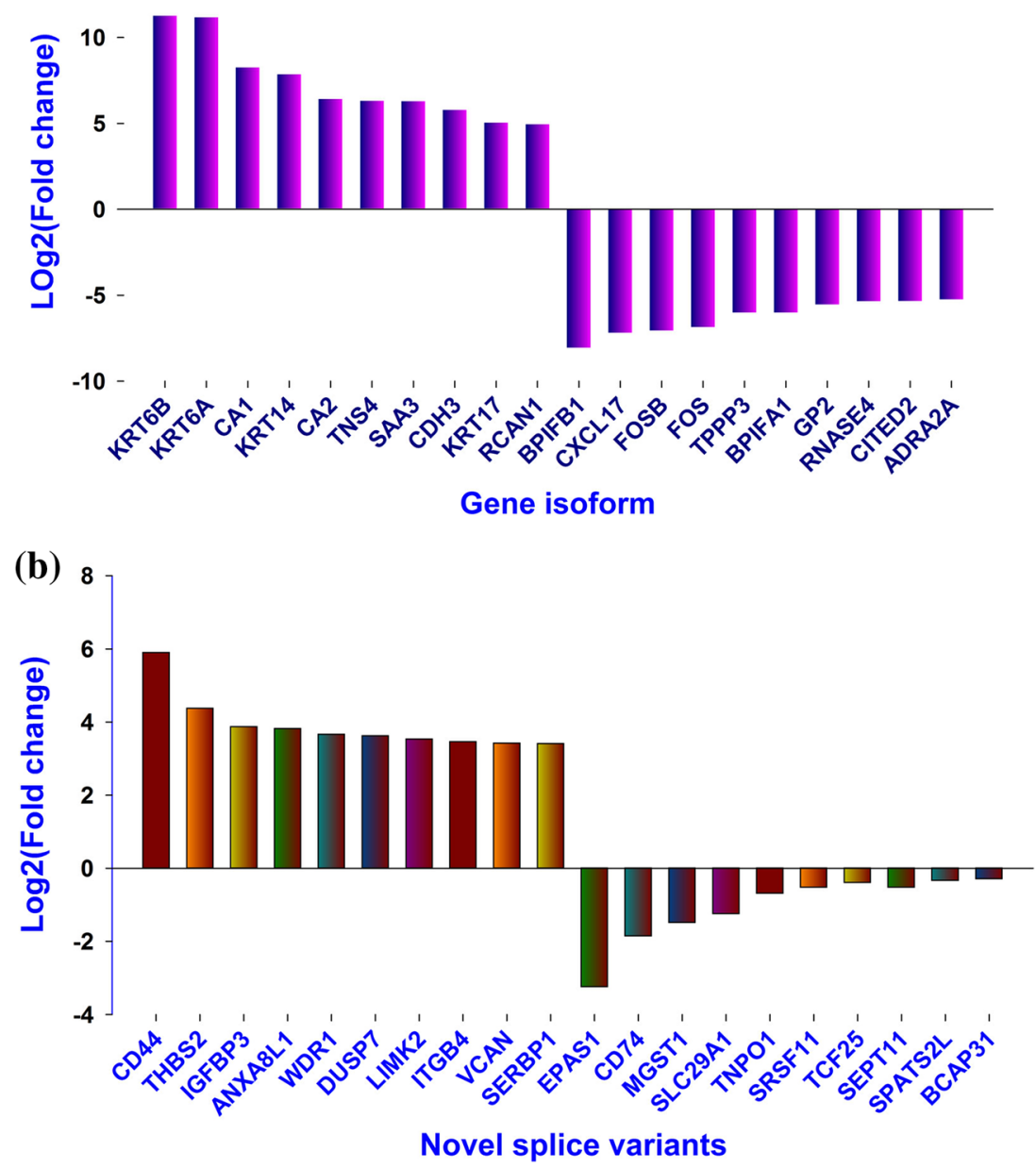

unravel the novel splice isoforms expressed in horn cancer as a result of complex RNA editing events through highthroughput transcriptome analyses of horn cancer and its normal counterpart. We found and validated several splice isoforms expressed in horn cancer that might play a role in progression of horn cancer. Our results show that highthroughput transcriptome sequencing along with cautious in silico and wet lab validation approaches can identify novel splice isoforms associated with cancer.

Our analyses with Cuffdiff for differential expression revealed several differentially expressed known isoforms in HC. Many of the gene isoforms identified are known to be involved in varieties of cancers; however, it would be important to discuss top deregulated and differentially expressed known gene isoforms in the present study. Amongst top up-regulated isoforms, KRT gene isoforms (KRT6A-11 fold, KRT6B-11 fold, KRT14-5 fold and $K R T 17-5$ fold) are expressed during differentiation of simple and stratified epithelial tissues, which justify their overexpression in horn cancer. These genes are also reported for overexpression in squamous cell carcinoma of lung (Hawthorn et al. 2006; Ohkura et al. 2005; Xue et al. 2011). At the second place, isoforms of $C A 1$ (8-fold) and CA2 (6.5-fold) genes were significantly overexpressed in HC. Both the gene isoforms are implicated in more advanced stage of the oral squamous cell carcinoma but not with metastasis (Liu et al. 2012). These isoforms might serve as a prospective biomarker to predict growth and metastasis of tumour cells. Next up-regulated isoform TNS4 variant (CTEN) is a member of family of proteins that are localized to integrin-linked focal adhesions. CTEN is implicated for increasing invasiveness through colony formation and anchorage-independent growth in colorectal, breast, gastric and pancreatic cancers (Liao et al. 2009; Sakashita et al. 2008; Albasri et al. 2011; Al-Ghamdi et al. 2013). SAA3 isoform which encodes for serum amyloid A protein has been widely studied as a marker in inflammation. Though its association in cancer is yet to be studied, it is observed that it might play a role in pre-metastatic stage and increases migration of metastatic cells to lungs (Hiratsuka et al. 2008). This observation makes SAA3 a plausible candidate target gene to track and prevent

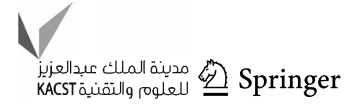


Table 1 List of pathways and functional categories deregulated in isoforms with significant differential expression in HC

\begin{tabular}{|c|c|c|}
\hline Pathway & Term & $P$ value \\
\hline \multicolumn{3}{|c|}{ Pathways deregulated in $\mathrm{HC}$} \\
\hline KEGG_PATHWAY & bta05200:Pathways in cancer & $9.63 \mathrm{E}-04$ \\
\hline KEGG_PATHWAY & bta05210:Colorectal cancer & $5.80 \mathrm{E}-03$ \\
\hline KEGG_PATHWAY & bta04010:MAPK signalling pathway & $2.26 \mathrm{E}-02$ \\
\hline KEGG_PATHWAY & bta04610:Complement and coagulation cascades & $2.33 \mathrm{E}-02$ \\
\hline KEGG_PATHWAY & bta00680:Methane metabolism & $5.11 \mathrm{E}-02$ \\
\hline \multicolumn{3}{|c|}{ Gene ontology categories deregulated in $\mathrm{HC}$} \\
\hline GOTERM_BP_FAT & GO:0001944 vasculature development & $1.39 \mathrm{E}-07$ \\
\hline GOTERM_BP_FAT & GO:0001568 blood vessel development & $1.21 \mathrm{E}-06$ \\
\hline GOTERM_BP_FAT & GO:0048514 blood vessel morphogenesis & $3.20 \mathrm{E}-06$ \\
\hline GOTERM_BP_FAT & GO:0001525 angiogenesis & $2.98 \mathrm{E}-05$ \\
\hline GOTERM_BP_FAT & GO:0008285 negative regulation of cell proliferation & $6.46 \mathrm{E}-05$ \\
\hline GOTERM_BP_FAT & GO:0060429 epithelium development & $1.02 \mathrm{E}-04$ \\
\hline GOTERM_MF_FAT & GO:0003700 transcription factor activity & $2.36 \mathrm{E}-04$ \\
\hline GOTERM_BP_FAT & GO:0035295 tube development & $2.73 \mathrm{E}-04$ \\
\hline GOTERM_BP_FAT & GO:0006357 regulation of transcription from RNA polymerase II promoter & $4.24 \mathrm{E}-04$ \\
\hline GOTERM_BP_FAT & GO:0010604 positive regulation of macromolecule metabolic process & $4.64 \mathrm{E}-04$ \\
\hline GOTERM_BP_FAT & GO:0030855 epithelial cell differentiation & $6.88 \mathrm{E}-04$ \\
\hline GOTERM_BP_FAT & GO:0008219 cell death & $7.15 \mathrm{E}-04$ \\
\hline GOTERM_BP_FAT & GO:0010628 positive regulation of gene expression & $7.84 \mathrm{E}-04$ \\
\hline GOTERM_BP_FAT & GO:0016265 death & $8.32 \mathrm{E}-04$ \\
\hline GOTERM_BP_FAT & GO:0009967 positive regulation of signal transduction & $9.19 \mathrm{E}-04$ \\
\hline
\end{tabular}

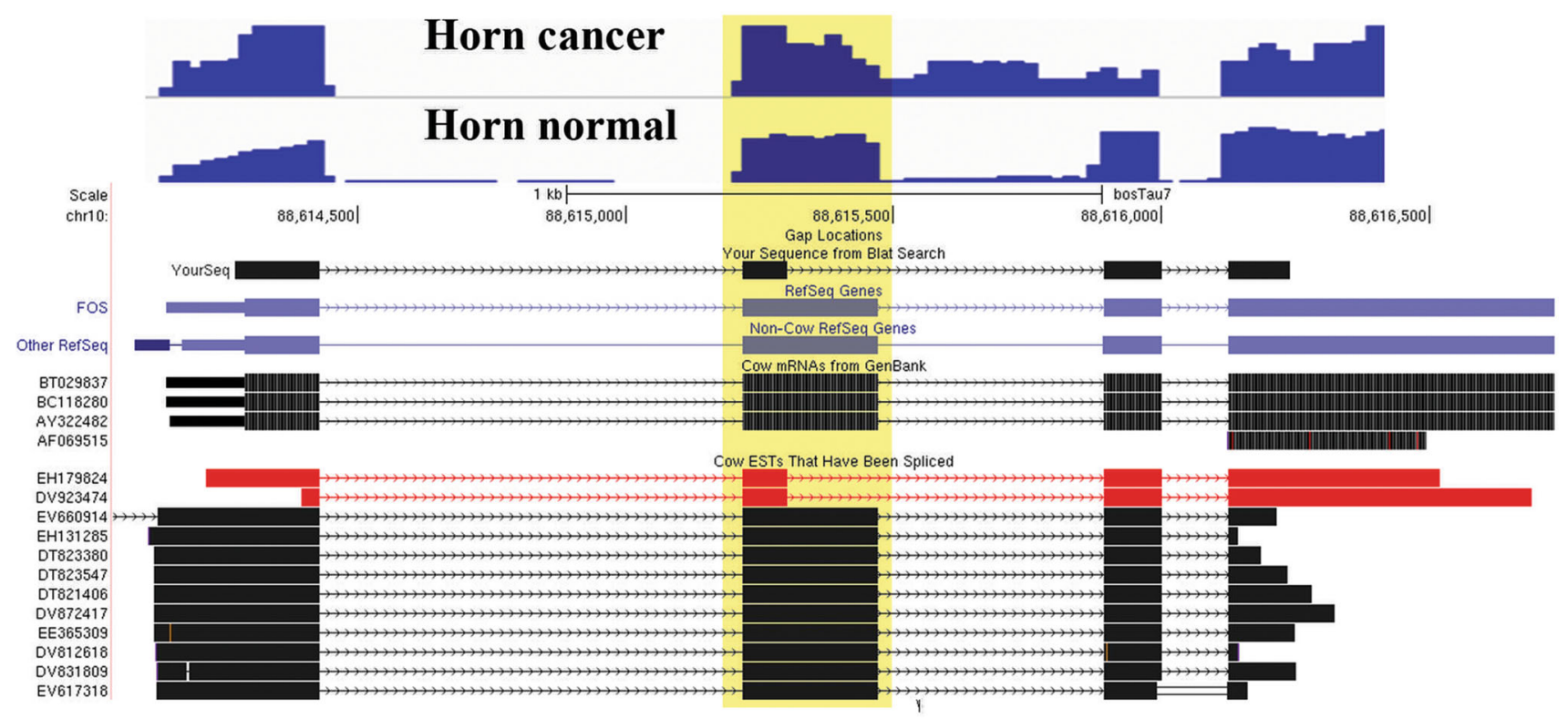

Fig. 2 Representative figure of typical novel splice variant identified in our study. Figure was reconstructed using images from IGV and UCSC genome browsers. At the top of the figure (blue peaks) coverage tracks from IGV genome viewer are shown, while the diagrammatic representation showing transcript structures for FOS gene was taken from UCSC genome browser after BLAT analysis of reads showing novel splice variation. Black blocks show exonic regions, while regions with arrows indicate intronic regions. ESTs supporting the splice variation are shown in red. The region marked with faint yellow block shows the exon with splice change 
Table 2 List of splice variants selected for validation along with type of variation and protein change

\begin{tabular}{|c|c|c|c|c|c|}
\hline Gene & $\begin{array}{l}\text { Accession no. of } \\
\text { reference gene }\end{array}$ & $\begin{array}{l}\text { EST } \\
\text { support }\end{array}$ & Type of variation & Chr. & Protein change \\
\hline FOS & NM_182786.2 & Yes & Alternate $3^{\prime}$ splicing exon 2 & 10 & Premature stop codon after 104 aa \\
\hline MAPK9 & NM_001046369.1 & Yes & Alternate $5^{\prime}$ splicing exon 10 & 7 & Premature stop codon after 293 aa \\
\hline FGFR1 & NM_001110207.1 & No & $\begin{array}{l}\text { Skipping of exon } 2 \text {, alternate } 3^{\prime} \\
\text { splicing exon } 3\end{array}$ & 27 & Deletion of aa 31-117, loss of Ig1_FGFR domain \\
\hline FGFR2 & NM_001205310.1 & No & Skipping of exon $10-13$ & 26 & Deletion of aa 448-639, loss of PTKc_FGFR domain \\
\hline MFAP4 & NM_001080217.1 & No & $\begin{array}{l}\text { skipping of exon } 2-5 \text {, alternate } 5^{\prime} \\
\text { splicing exon } 6\end{array}$ & 19 & $\begin{array}{l}\text { Premature stop codon after } 157 \text { aa, part of fibrinogen } \\
\text { related domain lost }\end{array}$ \\
\hline HSP90AB1 & NM_001079637.1 & Yes & Intron retention between exon $7-8$ & 23 & Premature stop codon after 380 aa, HSP90 domain lost \\
\hline RPS6KB1 & NM_205816.1 & No & Alternative $3^{\prime}$ splicing exon 7 & 19 & $\begin{array}{l}\text { Premature stop codon after } 234 \text { aa, part of STKc domain } \\
\text { lost }\end{array}$ \\
\hline CREBBP & NM_001164022.1 & No & Alternate $3^{\prime}$ splicing exon 9 & 25 & Premature stop codon after 649 aa, Creb domain lost \\
\hline CRK & NM_001192334.1 & Yes & $\begin{array}{l}\text { Novel exon between } 1-2 \text {, alternate } \\
3^{\prime} \text { splicing of exon } 2\end{array}$ & 19 & Premature stop codon after 204 aa, SH3 domain lost \\
\hline PTPN6 & NM_001098017.1 & No & Skipping of exon 12 & 5 & $\begin{array}{l}\text { Premature stop codon after } 457 \text { aa, part of PTPc domain } \\
\text { lost }\end{array}$ \\
\hline PTPRU & NM_001191494.1 & No & Alternate $5^{\prime}$ splicing exon 7 & 2 & $\begin{array}{l}\text { Premature stop codon after } 312 \text { aa, several domains of } \\
\text { fibronectine and PTPc are lost }\end{array}$ \\
\hline SUFU & NM_001098083.2 & No & Alternate exon 11 & 26 & $\begin{array}{l}\text { Same size of protein with different terminal } 52 \text { aa, } \\
\text { different } C \text { terminal domain than ref. }\end{array}$ \\
\hline FN1 & NM_001163778.1 & No & Skipping of exon 32 & 2 & Deletion of 1631-1720 aa without frame shift \\
\hline FN1 & NM_001163778.1 & Yes & Novel exon 25 & 2 & Addition of 91 aa after position 1266 without frame shift \\
\hline
\end{tabular}

metastasis. $\mathrm{CDH} 3$ isoform encoding for adhesion glycoprotein is a member of cadherin family responsible for cell-cell adhesion and reported for its overexpression in several cancers (Paredes et al. 2012). RCAN1 isoform encodes for protein regulator of calcineurin 1 and its overexpression inhibits migration of cancer cells (Espinosa et al. 2009). Amongst top down-regulated isoforms, BPIFB1 (8-fold) and BPIFA1 (6-fold) are members of a family of secreted proteins with unclear biological role and function, but it has been reported that these proteins are differentially expressed in lung diseases (Bingle et al. 2012). CXCL17 is a latest member of the C-X-C chemokine family proteins and aberrant expression of it promotes tumour progression through angiogenesis (Matsui et al. 2012). Next down-regulated isoforms FOS and FOSB (7fold) are members of FOS family of proteins involved in the regulation of cell proliferation, differentiation, and transformation. These proteins are extensively studied and their down-regulation is shown to promote tumour progression and metastasis (Kim et al. 2010). TPPP3 isoform, also known as p20, is a new member of the tubulin polymerization promoting protein (TPPP) family playing a role in microtubule assembly. TPPP3 protein is not reported in cancer, but its knockdown suppressed cell proliferation and induced cell cycle arrest in HeLa cells (Zhou et al. 2010). Three isoforms GP2, RNASE4 and ADRA2A amongst top down-regulated genes are not reported to be differentially expressed in cancer. CITED2 isoform encoding for $\mathrm{Cbp} /$ P300-Interacting Transactivator protein plays an important role in cell proliferation by responding to TGF- $\alpha$ induction and TGF- $\beta$ suppression to orchestrate cellular proliferation and quiescence, respectively. It is also reported that CITED2 knockdown caused tumour shrinkage and increased overall host mouse survival rates (Chou et al. 2012).

Gene ontology and pathway analyses of differentially expressed isoforms revealed important insights about the predominantly affected functions in $\mathrm{HC}$. Gene ontology suggested that $\mathrm{HC}$ is an actively growing tumour with changes involving epithelial cell differentiation and epithelium development with angiogenesis. Pathway analyses suggested that cancer pathways and MAPK signalling pathway responsible for uncontrolled cell growth are differentially enriched in HC. Further in-depth analysis of genes involved in MAPK/ERK signalling pathway is necessary to support and confirm their role in uncontrolled cell growth. Pathway analysis also resulted in clustering of pathways related to cardiomyopathy. Investigation of the genes (ACTN1, ACTN4, ADCY6, CTNNB1, DSP, EMD, ITGA2, ITGA5, ITGAV, ITGB1, ITGB3, ITGB5, JUP, PRKAA1, TPM1, TPM2, TPM4, and TCF7) clustered under the cardiomyopathy pathways revealed involvement of these genes in calcium binding pathways. As, calcium binding is important for normal functioning of heart 
Fig. 3 RT-qPCR analysis of novel splicing events. RT-qPCR analysis of novel splicing events on multiple horn cancer samples. The relative expression of reference (a) and variant (b) isoforms of candidate genes was analysed in four horn cancer tissue samples relative to four normal horn tissues. Gene names indicated below are the columns for respective genes, where _ $\mathrm{R}$ and _ $\mathrm{V}$ following gene names indicate reference and variant isoforms, respectively. The logarithmic transformation of relative quantitation $\left(\log _{2} R Q\right)$ of each candidate isoform in cancer tissues was plotted against their expression in normal tissue. Error bar indicates standard error of mean (SEM) of four biological replicates of $\mathrm{HC}$ or $\mathrm{HN}$
Horn Normal Horn Cancer
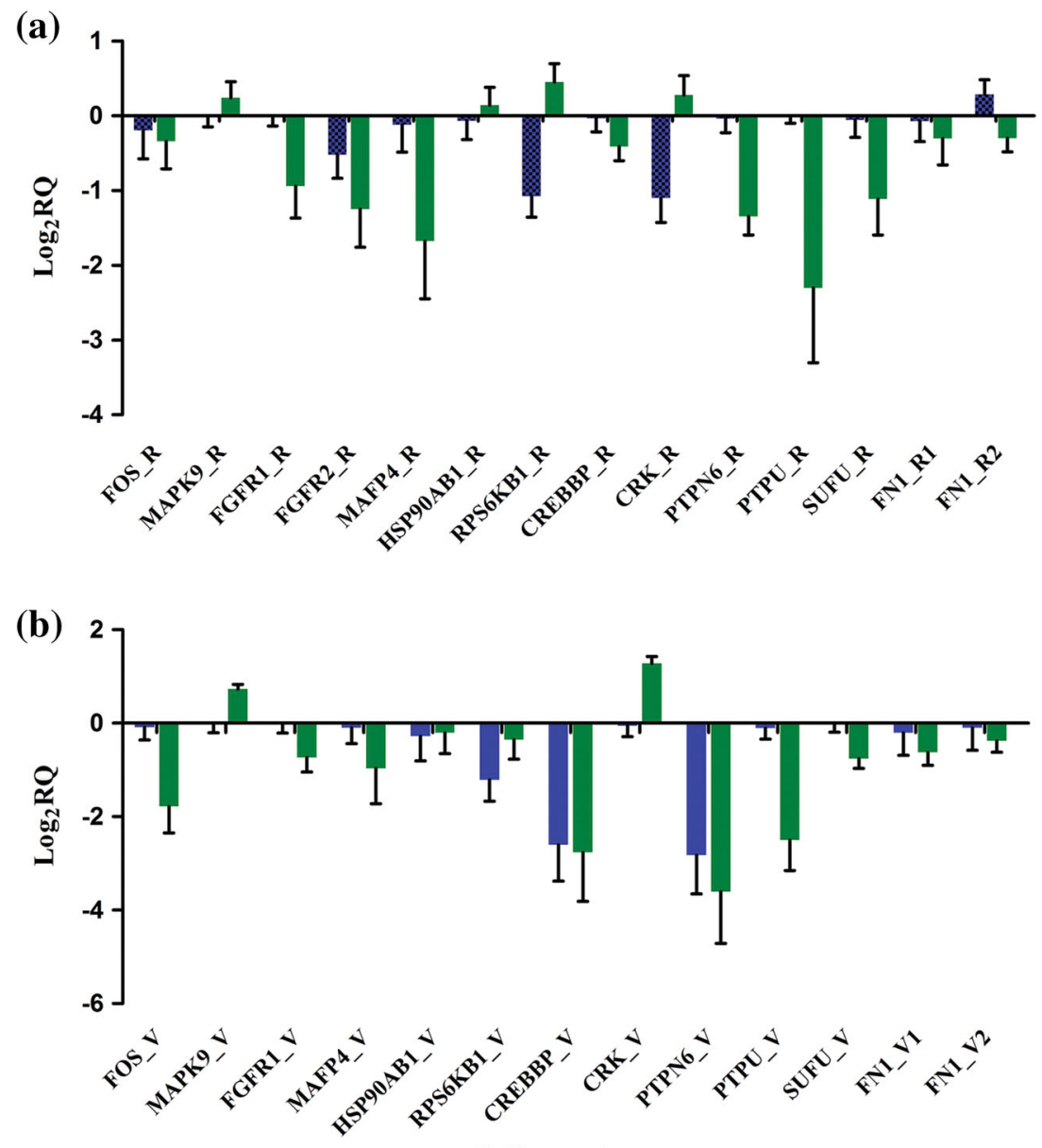

through binding with troponins, enrichment of these genes resulted in the enrichment of cardiomyopathy pathways (Farah and Reinach 1995).

Our analyses with Cuffcompare detected several transcripts with novel splicing amongst all detected transcripts classified as intergenic, complete match, novel or unknown. To reduce these novel splicing events to more meaningful conclusion and to reduce number of false positives, we employed rigorous bioinformatics analysis. As a first step, we reduced these numbers by selecting only those novel splice events which were clustered along with cancer pathways in pathway analysis; this helped us to select splicing events which might be associated with $\mathrm{HC}$. Second, we analysed all selected splicing events by UCSC genome browser, BLAT and IGV to check for EST support, to verify novel splicing and to eliminate splicing events not supported by reads, respectively. We also eliminated the events which were present at the end of the reads. As a third and final step, we performed blastp of the translated protein sequences of novel splices transcripts to examine amino acid changes in the sequence of the protein. In this step, we selected only splicing events which resulted in significant protein change or premature stop codon. Eventually, we selected 14 novel splicing events and confirmed their presence by end-point PCR and by RTqPCR. This intensive selection and filtering of novel splicing events made sure that only splice isoforms which might be significantly associated with HC are analysed.

Our validation of selected novel splicing events revealed discrepancy in the expression profiles obtained by RT-qPCR with that of RNA-Seq. The contrasting differential expression profile obtained with RT-qPCR in few isoforms reveals the limitation of RNA-Seq to identify reads originating from specific isoform and therefore requires additional validation using $\mathrm{RT}$-qPCR for changes in gene expression, while confirmation of almost all splicing events by RT-qPCR denotes reliability of RNASeq for high throughput detection of novel splicing events. Additionally, differential expression of all in silico selected splice isoforms across multiple cancer samples validates our approach for selection of novel splice isoforms relevant to cancer. We found a consistent pattern of down-regulation or up-regulation in both the reference and variant isoforms across all four samples, which 
emphasizes the possible development of these isoforms as biomarkers for horn cancer. Additionally, 9 out of 14 variants that we selected contain premature termination codon as a result of novel splicing; however, it requires further detailed investigation of effects produced by nonsense-mediated decay of these aberrant transcript on overall expression of the gene, if any.

Since horn cancer has been analysed previously for detection of novel splice variants (Patel et al. 2013), we sought to identify any differences or similarities between results, if any. Surprisingly, none of the previously identified and validated eight splice isoforms were detected in the present study. However, several reasons could be attributed for such an observation. As the previous study was a preliminary study involving small size of dataset without any quality filtering of the data, skipping of all the previous events identified is quite possible. Second, the present study identified and selected a set of novel splice isoforms whose probability of association with horn cancer is much more, as the events were identified in a logical and systematic way after enrichment of genes associated with horn cancer using GO analysis. Third, the software used for assembly (GS de novo assembler vs Cufflinks) and detection of splice events (Blat vs Cuffcompare) was different and it is important to note that full-length transcript assembly is very difficult, which is additionally complicated by artifacts in sample preparation, sequencing and read alignment. The above listed reasons and small-scale analysis without any type of selection criteria of previously generated dataset resulted in skipping of already identified novel splice isoforms. Nonetheless, the findings reported in the present study are absolutely novel and add to existing knowledge about splice isoforms in HC.

The 14 novel splicing events selected for verification might be considered as biomarkers as their potential effect on cancer progression. Consistent down-regulation of both the reference and variant was found for FOS gene by RTqPCR across four HC samples. Alternate $3^{\prime}$ splicing of exon2 in FOS resulted in premature stop codon just after position 104 aa culminating in loss of basic leucine zipper DNA-binding domain responsible for specific DNA binding. Although transcripts containing premature stop codon are subjected to nonsense-mediated decay (NMD), it has been studied that stress response elicited in tumour microenvironment leads to inhibition of NMD (Wang et al. 2011). Further studies are required to support such speculation and to study the effect of aberrant expression of this splice isoform on tumour progression. MAPK9 splice isoform which is a member of MAP kinase family of genes is also called as c-Jun N-terminal kinases (JNK2) involved in proliferation, differentiation, transcription regulation and development (Raciti et al. 2012). MAPK9 splice isoform exhibited premature stop codon after 293 aa and loss of domain responsible for serine/threonine kinase activity. We observed consistent up-regulation of both the reference and variant for MAPK9 gene by RT-qPCR across four $\mathrm{HC}$ samples. FGFRI and FGFR2 are the members of the fibroblast growth factor receptor (FGFR) family of genes with a variety of roles in cancer (Holzmann et al. 2012). Splice variation in FGFRI led to loss of IG1 domain which leads to activation of distinct signalling cascades (Zhang et al. 2006), while splice change observed in FGFR2 with loss of PTKc domain could not be validated with RT-PCR. Both the genes FGFRl and FGFR2, reference and variant, were found to be down-regulated by RT-qPCR. MFAP4 encodes for bovine microfibril-associated protein with probable role in calcium-dependent cell adhesion or intercellular interactions. We also observed intron retention in $H S P 90 A B 1$ transcript resulting in premature termination after 380 aa position. $H S P 90 A B 1$ plays an important role in folding newly synthesized proteins or stabilizing and refolding denatured proteins after stress (Cheng et al. 2012). RPS6KB1 splice isoform is part of a protein kinase family involved in the regulation of cellular growth, insulin, and cellular energy. Overexpression of RPS6KB1 plays an important role in hepatocellular carcinoma ( $\mathrm{Li}$ et al. 2012) and correlates with adverse prognosis. Similarly, CREBBP (Mullighan et al. 2011), CRK (Sriram and Birge 2010), PTPN6 and PTPRU (Hoekstra et al. 2012) genes which might play a probable role in cancer revealed premature termination with loss of domains related to function as shown in Table 2. SUFU gene encodes negative regulator of Hedgehog signalling pathway exhibiting signalling cascade that plays a role in pattern formation and cellular proliferation during development. The gene is not well studied in the bovine, but two isoforms with different $\mathrm{c}$ terminal have been reported in humans (Tostar et al. 2012). $S U F U$ splice isoform identified in this study encodes for longer isoform with different $\mathrm{C}$ terminal than reference. FN1 encodes fibronectin, a glycoprotein present at the cell surface and in extracellular matrix and is involved in cell adhesion and migration processes including embryogenesis, wound healing, blood coagulation, host defense, and metastasis (Goossens et al. 2009). Splice variation of novel exon 25 and skipping of exon 32 revealed in this isoform and resulted in varying length of protein without frameshift. In total, we confirmed expression of these 13 novel splice isoforms, however it remains to be investigated whether these putative protein isoforms are indeed translated in to proteins and in turn functional consequences of these variations. Furthermore, we found an interesting aspect of our analysis of AS events in $\mathrm{HC}$ as majority of novel splice isoforms supported by ESTs reported in bovine foetus. This may be due to the activation of genes or isoforms taking part in the foetal development by carcinogenic genes. This observation is supported by an 
earlier study showing antigenic similarity between bovine horn cancer and foetal tissue (Kuchroo et al. 1979).

\section{Conclusion}

The results reported here are novel in terms of alternative splicing prevailing in $\mathrm{HC}$ and have potential implications for intervention of $\mathrm{HC}$ at molecular level. Herein, we reported and confirmed several novel splice isoforms differentially expressed in $\mathrm{HC}$ which might be useful as biomarkers to diagnose the $\mathrm{HC}$ at early stage. Rigorous and cautious filtering of several novel splice isoforms resulted in splice isoforms, most probably associated with HC. In summary, we have identified and validated fourteen putative novel isoforms which are significantly associated with $\mathrm{HC}$ and discussed their possible involvement in cancer progression. Further extensive follow-up analysis of novel splice isoforms found in this study might be useful in the development of prospective intervention strategies for $\mathrm{HC}$. The results reported here might also be helpful to understand other cancers prevalent in other species and refine the available information on transcriptome repertoire of bovine species.

Acknowledgements The authors would like to acknowledge the contribution of Gujarat State Biotechnology Mission for providing financial assistance to carry out the research work. The authors are also thankful to Dr. Uday Koringa, Dr. P. B. Patel and Banas Dairy for providing horn cancer and normal tissues for comparative study.

\section{Compliance with ethical standards}

Conflict of interest The authors declare that they have "no competing interests'.

Open Access This article is distributed under the terms of the Creative Commons Attribution 4.0 International License (http:// creativecommons.org/licenses/by/4.0/), which permits unrestricted use, distribution, and reproduction in any medium, provided you give appropriate credit to the original author(s) and the source, provide a link to the Creative Commons license, and indicate if changes were made.

\section{References}

Albasri A, Aleskandarany M, Benhasouna A, Powe DG, Ellis IO, Ilyas M, Green AR (2011) CTEN (C-terminal tensin-like), a novel oncogene overexpressed in invasive breast carcinoma of poor prognosis. Breast Cancer Res Treat 126(1):47-54. doi:10. 1007/s10549-010-0890-3

Al-Ghamdi S, Cachat J, Albasri A, Ahmed M, Jackson D, Zaitoun A, Guppy N, Otto WR, Alison MR, Kindle KB, Ilyas M (2013) C-terminal tensin-like gene functions as an oncogene and promotes cell motility in pancreatic cancer. Pancreas 42(1):135-140. doi:10.1097/MPA.0b013e3182557ceb
Altschul SF, Gish W, Miller W, Myers EW, Lipman DJ (1990) Basic local alignment search tool. J Mol Biol 215(3):403-410. doi:10. 1016/S0022-2836(05)80360-2

Bingle CD, Wilson K, Lunn H, Barnes FA, High AS, Wallace WA, Rassl D, Campos MA, Ribeiro M, Bingle L (2010) Human LPLUNC1 is a secreted product of goblet cells and minor glands of the respiratory and upper aerodigestive tracts. Histochem Cell Biol 133(5):505-515. doi:10.1007/s00418-010-0683-0

Bingle L, Wilson K, Musa M, Araujo B, Rassl D, Wallace WA, LeClair EE, Mauad T, Zhou Z, Mall MA, Bingle CD (2012) BPIFB1 (LPLUNC1) is upregulated in cystic fibrosis lung disease. Histochem Cell Biol 138(5):749-758. doi:10.1007/ s00418-012-0990-8

Burggraaf H (1935) Kanker aan de basis van de hoorns bij zebus. T Diergeneesk 62:1121-1136

Chattopadhyay SK, Jandrotia VS, Ramkumar Iyer PKR (1982) Horn cancer in sheep. Indian Vet J 59:319-320

Cheng Q, Chang JT, Geradts J, Neckers LM, Haystead T, Spector NL, Lyerly HK (2012) Amplification and high-level expression of heat shock protein 90 marks aggressive phenotypes of human epidermal growth factor receptor 2 negative breast cancer. Breast Cancer Res 14(2):R62. doi:10.1186/bcr3168

Chou YT, Hsieh CH, Chiou SH, Hsu CF, Kao YR, Lee CC, Chung CH, Wang YH, Hsu HS, Pang ST, Shieh YS, Wu CW (2012) CITED2 functions as a molecular switch of cytokine-induced proliferation and quiescence. Cell Death Differ 19(12):2015-2028. doi:10.1038/cdd.2012.91

Damodaran S, Sundararaj A, Ramakrishnan R (1979) Horn cancer in bulls. Indian Vet J 56(3):248-249

Dreszer TR, Karolchik D, Zweig AS, Hinrichs AS, Raney BJ, Kuhn RM, Meyer LR, Wong M, Sloan CA, Rosenbloom KR, Roe G, Rhead B, Pohl A, Malladi VS, Li CH, Learned K, Kirkup V, Hsu F, Harte RA, Guruvadoo L, Goldman M, Giardine BM, Fujita PA, Diekhans M, Cline MS, Clawson H, Barber GP, Haussler D, James Kent W (2012) The UCSC Genome Browser database: extensions and updates 2011. Nucleic Acids Res 40(Database Issue):D918-D923. doi:10.1093/nar/gkr1055

Erho N, Buerki C, Triche TJ, Davicioni E, Vergara IA (2012) Transcriptome-wide detection of differentially expressed coding and non-coding transcripts and their clinical significance in prostate cancer. J Oncol 2012:541353. doi:10.1155/2012/541353

Espinosa AV, Shinohara M, Porchia LM, Chung YJ, McCarty S, Saji M, Ringel MD (2009) Regulator of calcineurin 1 modulates cancer cell migration in vitro. Clin Exp Metastasis 26(6):517-526. doi:10.1007/s10585-009-9251-1

Farah CS, Reinach FC (1995) The troponin complex and regulation of muscle contraction. FASEB J 9(9):755-767

Goossens K, Van Soom A, Van Zeveren A, Favoreel H, Peelman LJ (2009) Quantification of fibronectin 1 (FN1) splice variants, including two novel ones, and analysis of integrins as candidate FN1 receptors in bovine preimplantation embryos. BMC Dev Biol 9:1. doi:10.1186/1471-213X-9-1

Gupta RK, Sadana JR, Kuchroo VK, Kalra DS (1980) Horn cancer in an intact bull. Vet Rec 107(13):312

Hawthorn L, Stein L, Panzarella J, Loewen GM, Baumann H (2006) Characterization of cell-type specific profiles in tissues and isolated cells from squamous cell carcinomas of the lung. Lung Cancer 53(2):129-142. doi:10.1016/j.lungcan.2006.04.015

Hiraoka N, Yamazaki-Itoh R, Ino Y, Mizuguchi Y, Yamada T, Hirohashi S, Kanai Y (2011) CXCL17 and ICAM2 are associated with a potential anti-tumor immune response in early intraepithelial stages of human pancreatic carcinogenesis. Gastroenterology 140(1):310-321. doi:10.1053/j.gastro.2010.10.009

Hiratsuka S, Watanabe A, Sakurai Y, Akashi-Takamura S, Ishibashi S, Miyake K, Shibuya M, Akira S, Aburatani H, Maru Y (2008) The S100A8-serum amyloid A3-TLR4 paracrine cascade 
establishes a pre-metastatic phase. Nat Cell Biol 10(11):1349-1355. doi:10.1038/ncb1794

Hoekstra E, Peppelenbosch MP, Fuhler GM (2012) The role of protein tyrosine phosphatases in colorectal cancer. Biochim Biophys Acta 1826(1):179-188. doi:10.1016/j.bbcan.2012.04. 001

Holzmann K, Grunt T, Heinzle C, Sampl S, Steinhoff H, Reichmann N, Kleiter M, Hauck M, Marian B (2012) Alternative splicing of fibroblast growth factor receptor IgIII loops in cancer. J Nucleic Acids 2012:950508. doi:10.1155/2012/950508

Huang DW, Sherman BT, Lempicki RA (2009) Systematic and integrative analysis of large gene lists using DAVID bioinformatics resources. Nat Protoc 4(1):44-57. doi:10.1038/nprot. 2008.211

Irimia M, Blencowe BJ (2012) Alternative splicing: decoding an expansive regulatory layer. Curr Opin Cell Biol 24(3):323-332. doi:10.1016/j.ceb.2012.03.005

Jakhesara SJ, Koringa PG, Bhatt VD, Shah TM, Vangipuram S, Shah S, Joshi CG (2013a) RNA-Seq reveals differentially expressed isoforms and novel splice variants in buccal mucosal cancer. Gene 516(1):24-32. doi:10.1016/j.gene.2012.11.079

Jakhesara SJ, Koringa PG, Joshi CG (2013b) Identification of novel exons and transcripts by comprehensive RNA-Seq of horn cancer transcriptome in Bos indicus. J Biotechnol 165(1):37-44. doi:10.1016/j.jbiotec.2013.02.015

Joshi BP, Soni PB, Fefar DT, Ghodasara DJ, Prajapati KS (2009) Epidemiological and pathological aspects of horn cancer in cattle of Gujarat. Indian J Field Vet 5(2):15-18

Kim JH, Lee JY, Lee KT, Lee JK, Lee KH, Jang KT, Heo JS, Choi SH, Rhee JC (2010) RGS16 and FosB underexpressed in pancreatic cancer with lymph node metastasis promote tumor progression. Tumour Biol 31(5):541-548. doi:10.1007/s13277010-0067-z

Koringa PG, Jakhesara SJ, Bhatt VD, Meshram CP, Patel AK, Fefar DT, Joshi CG (2013) Comprehensive transcriptome profiling of squamous cell carcinoma of horn in Bos indicus. Vet Comp Oncol. doi:10.1111/vco.12079

Kuchroo V, Gupta RK, Kalra DS (1979) Antigenic similarity between squamous cell carcinoma of horn (horn cancer) and normal bovine foetal tissues. Trop Anim Health Prod 11(4):203-207

Kulkarni HV (1953) Carcinoma of horn in bovines of old Baroda state. Indian Vet J 29:415-421

Li H, Handsaker B, Wysoker A, Fennell T, Ruan J, Homer N, Marth G, Abecasis G, Durbin R (2009) The sequence alignment/map format and SAMtools. Bioinformatics 25(16):2078-2079. doi:10.1093/bioinformatics/btp352

Li PD, Zhang WJ, Zhang MY, Yuan LJ, Cha YL, Ying XF, Wu G, Wang HY (2012) Overexpression of RPS6KB1 predicts worse prognosis in primary HCC patients. Med Oncol 29(5):3070-3076. doi:10.1007/s12032-012-0268-y

Li L, Liu J, Yu W, Lou X, Huang B, Lin B (2013) Deep transcriptome profiling of ovarian cancer cells using next-generation sequencing approach. Methods Mol Biol 1049:139-169. doi:10.1007/ 978-1-62703-547-7_12

Liao YC, Chen NT, Shih YP, Dong Y, Lo SH (2009) Up-regulation of $\mathrm{C}$-terminal tensin-like molecule promotes the tumorigenicity of colon cancer through beta-catenin. Cancer Res 69(11):4563-4566. doi:10.1158/0008-5472.CAN-09-0117

Liu CM, Lin YM, Yeh KT, Chen MK, Chang JH, Chen CJ, Chou MY, Yang SF, Chien MH (2012) Expression of carbonic anhydrases I/II and the correlation to clinical aspects of oral squamous cell carcinoma analyzed using tissue microarray. J Oral Pathol Med 41(7):533-539. doi:10.1111/j.1600-0714.2012.01135.x

Matsui A, Yokoo H, Negishi Y, Endo-Takahashi Y, Chun NA, Kadouchi I, Suzuki R, Maruyama K, Aramaki Y, Semba K, Kobayashi E, Takahashi M, Murakami T (2012) CXCL17 expression by tumor cells recruits CD11b + Gr1 high F4/80cells and promotes tumor progression. PLoS One 7(8):e44080. doi:10.1371/journal.pone.0044080

Mullighan CG, Zhang J, Kasper LH, Lerach S, Payne-Turner D, Phillips LA, Heatley SL, Holmfeldt L, Collins-Underwood JR, Ma J, Buetow KH, Pui CH, Baker SD, Brindle PK, Downing JR (2011) CREBBP mutations in relapsed acute lymphoblastic leukaemia. Nature 471(7337):235-239. doi:10.1038/ nature 09727

Ohkura S, Kondoh N, Hada A, Arai M, Yamazaki Y, Sindoh M, Takahashi M, Matsumoto I, Yamamoto M (2005) Differential expression of the keratin-4, $-13,-14,-17$ and transglutaminase 3 genes during the development of oral squamous cell carcinoma from leukoplakia. Oral Oncol 41(6):607-613. doi:10.1016/j. oraloncology.2005.01.011

Paredes J, Figueiredo J, Albergaria A, Oliveira P, Carvalho J, Ribeiro AS, Caldeira J, Costa AM, Simoes-Correia J, Oliveira MJ, Pinheiro H, Pinho SS, Mateus R, Reis CA, Leite M, Fernandes MS, Schmitt F, Carneiro F, Figueiredo C, Oliveira C, Seruca R (2012) Epithelial E- and P-cadherins: role and clinical significance in cancer. Biochim Biophys Acta 1826(2):297-311. doi:10.1016/j.bbcan.2012.05.002

Patel AK, Bhatt VD, Tripathi AK, Sajnani MR, Jakhesara SJ, Koringa PG, Joshi CG (2013) Identification of novel splice variants in horn cancer by RNA-Seq analysis in Zebu cattle. Genomics 101(1):57-63. doi:10.1016/j.ygeno.2012.10.001

Raciti M, Lotti LV, Valia S, Pulcinelli FM, Di Renzo L (2012) JNK2 is activated during ER stress and promotes cell survival. Cell Death Dis 3:e429. doi: $10.1038 /$ cddis.2012.167

Rezende AML, Naves PT (1975) Horn core cancer in a zebu cow, imported to Brazil. Pesq Agropec Bras Série Veterinária $10: 41-44$

Roberts A, Pimentel H, Trapnell C, Pachter L (2011) Identification of novel transcripts in annotated genomes using RNA-Seq. Bioinformatics 27(17):2325-2329. doi:10.1093/bioinformatics/btr355

Sakashita K, Mimori K, Tanaka F, Kamohara Y, Inoue H, Sawada T, Hirakawa K, Mori M (2008) Prognostic relevance of Tensin4 expression in human gastric cancer. Ann Surg Oncol 15(9):2606-2613. doi:10.1245/s10434-008-9989-8

Sriram G, Birge RB (2010) Emerging roles for crk in human cancer. Genes Cancer 1(11):1132-1139. doi:10.1177/ 1947601910397188

Thorvaldsdottir H, Robinson JT, Mesirov JP (2012) Integrative Genomics Viewer (IGV): high-performance genomics data visualization and exploration. Brief Bioinform. doi:10.1093/ $\mathrm{bib} / \mathrm{bbs} 017$

Tostar U, Finta C, Rahman MF, Shimokawa T, Zaphiropoulos PG (2012) Novel mechanism of action on Hedgehog signaling by a suppressor of fused carboxy terminal variant. PLoS One 7(5):e37761. doi:10.1371/journal.pone.0037761

Trapnell C, Williams BA, Pertea G, Mortazavi A, Kwan G, van Baren MJ, Salzberg SL, Wold BJ, Pachter L (2010) Transcript assembly and quantification by RNA-Seq reveals unannotated transcripts and isoform switching during cell differentiation. Nat Biotechnol 28(5):511-515. doi:10.1038/nbt.1621

Tripathi AK, Koringa PG, Jakhesara SJ, Ahir VB, Ramani UV, Bhatt VD, Sajnani MR, Patel DA, Joshi AJ, Shanmuga SJ, Rank DN, Joshi CG (2012) A preliminary sketch of horn cancer transcriptome in Indian zebu cattle. Gene 493(1):124-131. doi:10.1016/j. gene.2011.11.007

Udharwar SV, Aher VD, Yadav GU, Bhikane AU, Dandge BP (2008) Study on incidence, predisposing factors, symptomatology and treatment of horn cancer in bovine with special reference to surgery and chemotherapy. Vet World 1(1):7-9

Wang D, Zavadil J, Martin L, Parisi F, Friedman E, Levy D, Harding H, Ron D, Gardner LB (2011) Inhibition of nonsense-mediated 
RNA decay by the tumor microenvironment promotes tumorigenesis. Mol Cell Biol 31(17):3670-3680. doi:10.1128/MCB. 05704-11

Wu TD, Watanabe CK (2005) GMAP: a genomic mapping and alignment program for mRNA and EST sequences. Bioinformatics 21(9):1859-1875. doi:10.1093/bioinformatics/bti310

Xue LY, Zou SM, Zheng S, Liu XY, Wen P, Yuan YL, Lin DM, Lu N (2011) Expressions of the gamma2 chain of laminin-5 and secreted protein acidic and rich in cysteine in esophageal squamous cell carcinoma and their relation to prognosis. Chin $\mathrm{J}$ Cancer 30(1):69-78

Ye J, Coulouris G, Zaretskaya I, Cutcutache I, Rozen S, Madden TL (2012) Primer-BLAST: a tool to design target-specific primers for polymerase chain reaction. BMC Bioinform 13:134. doi:10. 1186/1471-2105-13-134

Zhang P, Greendorfer JS, Jiao J, Kelpke SC, Thompson JA (2006) Alternatively spliced FGFR-1 isoforms differentially modulate endothelial cell activation of c-YES. Arch Biochem Biophys 450(1):50-62. doi:10.1016/j.abb.2006.03.017

Zhou W, Wang X, Li L, Feng X, Yang Z, Zhang W, Hu R (2010) Depletion of tubulin polymerization promoting protein family member 3 suppresses HeLa cell proliferation. Mol Cell Biochem 333(1-2):91-98. doi:10.1007/s11010-009-0208-0

Zubaidy AJ (1976) Horn cancer in cattle in Iraq. Vet Pathol $13: 435-454$ 